\title{
ArcheoSciences
}

Revue d'archéométrie

$30 \mid 2006$

Varia

\section{Arlette Leroi-Gourhan (1913-2005) : l'initiatrice de la palynologie appliquée à l'archéologie préhistorique}

Aline Emery-Barbier, Chantal Leroyer et Philippe Soulier

\section{(2) OpenEdition}

\section{Journals}

Édition électronique

URL : http://journals.openedition.org/archeosciences/411

DOI : 10.4000/archeosciences. 411

ISBN : 978-2-7535-1595-6

ISSN : 2104-3728

\section{Éditeur}

Presses universitaires de Rennes

\section{Édition imprimée}

Date de publication : 31 décembre 2006

Pagination : 227-23031 décembre 200631 décembre 2008

ISBN : 978-2-7535-0456-1

ISSN : $1960-1360$

\section{Référence électronique}

Aline Emery-Barbier, Chantal Leroyer et Philippe Soulier, « Arlette Leroi-Gourhan (1913-2005) :

l'initiatrice de la palynologie appliquée à l'archéologie préhistorique », ArcheoSciences [En ligne],

30 | 2006, document 22, mis en ligne le 22 juin 2009, consulté le 01 mai 2019. URL : http://

journals.openedition.org/archeosciences/411; DOI : 10.4000/archeosciences.411 


\title{
Arlette Leroi-Gourhan (1913-2005) : l'initiatrice de la palynologie appliquée à l'archéologie préhistorique
}

\author{
Aline Emery-Barbier*, Chantal Leroyer**, Philippe Soulier*
}

Arlette Leroi-Gourhan est décédée le 25 avril 2005 en son domicile familial de Vermenton.

Née Royer le 9 janvier 1913 à Paris, elle est issue d'une famille d'industriels. Son appartenance à une bourgeoisie aisée lui ouvre les portes des milieux intellectuels et artistiques. Elle pratique ainsi danse, musique et différents sports (natation, équitation, ski...) tandis qu'elle voyage beaucoup en Europe et en Afrique du Nord. Diplômée de l'École du Louvre, elle suit en 1934 les cours que l'ethnologue Marcel Mauss donne à l'EHESS. Elle y rencontre André LeroiGourhan et prend connaissance des travaux de rénovation des salles du musée d'Ethnologie du Trocadéro, qui fera place au musée de l'Homme en 1937. Comme bien des étudiants de cette période féconde, elle s'investit alors bénévolement dans ce travail de muséologie car le matériel afflue au musée. Elle participe ainsi à la réception des objets issus des célèbres et médiatiques " missions Citroën " d'Afrique et d'Asie, des missions d'observation et de collecte « DakarDjibouti » de Marcel Griaule et des travaux de Germaine Dieterlin. L'art africain étant alors particulièrement apprécié des milieux artistiques parisiens, de nombreuses expositions sont présentées au Musée, en particulier sur le Maghreb, le Sahara et l'Afrique sub-saharienne... De ces années d'élaboration du musée de l'Homme, elle conservera le souvenir d'un contexte dynamique et stimulant, qu'elle évoquera fréquemment et avec passion. Du déclin puis de la disparition organisée de ce Musée surgiront des regrets infinis qui rendront plus sombres les dernières années de sa vie.

Elle épouse André Leroi-Gourhan en 1936 et le couple part au Japon dès février 1937, André Leroi-Gourhan ayant obtenu une mission ethnographique de deux ans, financée par le gouvernement japonais. À la rentrée universitaire, il obtient une charge d'enseignement à l'Institut franco-japonais de Kyoto et Arlette Leroi-Gourhan s'associe alors entièrement au travail de son époux : elle réalise les travaux photographiques ainsi que le secrétariat, l'accompagnant en permanence lors de ses voyages hors de Kyoto. Ils consacrent ainsi l'été 1938 à l'étude des derniers Aïnous de lî̀le d'Hokkaïdo dans le nord de l'archipel nippon. Une part de la documentation amassée figurera dans la thèse de Lettres d'André Leroi-Gourhan " Archéologie du Pacifique nord », soutenue en juin 1944 mais l'intégralité des données collectées ne sera accessible qu'en 1989. Septuagénaire, Arlette Leroi-Gourhan publie alors, sous leurs deux noms, le récit de leur expérience (Leroi-Gourhan Arl., Leroi-Gourhan A., 1989), qui sera aussi diffusé au Japon en 1991 avec le concours du Professeur Ichiro Yamanaka (LeroiGourhan Arl., Yamanaka I., 1991).

La dégradation de la situation internationale va interrompre leur mission. À la suite des accords de Munich, Arlette Leroi-Gourhan quitte prématurément le Japon pour la France avec un premier chargement des objets destinés au musée de l'Homme ainsi qu'au musée Guimet. Elle consacre les quinze

\footnotetext{
*UMR 7041 / ArScAn, Maison de l'Archéologie et de l'Ethnologie, 92023 Nanterre cedex. (aline.emery-barbier@mae.u-paris10.fr)(philippe.soulier@ mae.u-paris10.fr)

** Ministère de la Culture, UMR 6566, Centre National de Préhistoire, 24000 Périgueux. (chantal.leroyer@culture.gouv.fr)
} 
années suivantes à l'éducation de ses quatre enfants et à la carrière professionnelle de son époux. Elle l'assiste comme au Japon dans les travaux administratifs, liés à ses charges d'enseignement à l'Université de Lyon et au musée de l'Homme.

À partir de 1954, Arlette Leroi-Gourhan va s'investir personnellement dans la recherche. Dans le cadre de l'école de fouille d'André Leroi-Gourhan, installée à Arcy-sur-Cure (Yonne) depuis 1949, elle va aborder un domaine alors à peine envisagé en archéologie : l'étude palynologique des dépôts préhistoriques en grotte. De ses échanges avec les préhistoriens, elle a acquis la certitude que l'approche environnementale est indispensable à la démarche archéologique et elle s'informe sur les avancées de la palynologie en Europe septentrionale. Ses connaissances l'entraînent à faire quelques prélèvements suivis d'analyses car elle se forme à la morphologie pollinique auprès de Madeleine Van Campo. Ses recherches vont s'accélérer à partir de 1955 avec la mise à disposition de locaux au musée de l'Homme pour installer un laboratoire. Avec les conseils de Madeleine Van Campo, Arlette Leroi-Gourhan poursuit ses premières expériences dans les grottes d'Arcy-sur-Cure mais aussi de Saint-Marcel (Indre), puis sur le site de la Cotte de Saint-Brelade (Jersey). Les résultats préliminaires des analyses d'Arcy sont publiés en 1956 (Van Campo et Leroi-Gourhan, 1956a et b).

Les premières contributions d'Arlette Leroi-Gourhan sont méthodologiques. Dans la séance de la SPF de juin 1955, elle expose ses premiers travaux sur les corrélations entre données palynologiques, datations C14 et identifications « culturelles » (1956a). En juillet 1956, au Congrès Préhistorique de Poitiers-Angoulême, elle souligne qu'au-delà de l'intérêt botanique, géologique ou préhistorique des analyses, leur aspect novateur concerne aussi l'" ethnologie préhistorique », car « la reconstitution du paysage botanique rend directement perceptible l'existence matérielle des hommes » (1956b).

Bientôt, la création d'un poste de technicien, dont bénéficie Michel Girard, et l'amélioration du protocole d'extraction que celui-ci met au point, permettent d'étendre les analyses en terrains secs. Arlette Leroi-Gourhan peut ainsi poursuivre les travaux engagés à Arcy-sur-Cure et publier avec son époux la première synthèse sur la chronologie quaternaire de ces grottes (Leroi-Gourhan Arl., Leroi-Gourhan A., 1965). Elle étend aussi son activité avec une diversification de ses champs d'investigation vers l'Afrique du Nord et le MoyenOrient $(1957,1958,1961$ a) et la multiplication des études de grottes françaises et espagnoles du Paléolithique supérieur : Isturitz (1959), Lascaux (1962), la Cueva del Otero (1966a), la Vache (1967a), l'abri Fritsch (1967b et c)... Dès 1959, au Congrès Préhistorique de France à Monaco, elle présente une vaste synthèse allant du Moustérien à l'Azilien (1960). Les études palynologiques vont désormais faire par- tie de la recherche préhistorique, complétant les données de la sédimentologie et de la paléontologie, tant pour les attributions chrono-climatiques des niveaux stratigraphiques que pour le repérage des perturbations biologiques ou anthropiques dans ces mêmes niveaux.

Les grands axes de ses recherches sont ainsi mis en place. Son souci premier reste toujours la valorisation des études palynologiques de sédiments archéologiques (1956b, 1963, 1965a, 1973a) - avec très tôt la prise de conscience de l'interdisciplinarité (1956a, 1966b, 1989a, 1992). En parallèle, ses nombreuses études de grottes du Paléolithique supérieur, en France et en Espagne (Isturitz, Lascaux, la Vache, Enlène, l'abri Fritsch, la Cueva del Otero, Tursac, le Portel, Pech Merle, La Cueva Morin, La Cueva del Pendo, Pégourié, la Riera, Saint Césaire) la mènent à proposer, à argumenter, et à préciser à plusieurs reprises une chronologie climatique du Paléolithique supérieur (1961b, 1965b, 1968a, 1973b, 1980a, 1984a, 1989b, 1997, Leroi- Gourhan et Girard, 1976 et 1979; Leroi-Gourhan et Renault-Miskovsky, 1977; Renault-Miskovsky et Leroi-Gourhan, 1981).

Par ailleurs, son important investissement au Moyen-Orient la conduit à y développer un autre domaine de recherche. Ses travaux sur le Levant s'organisent selon différentes problématiques : l'environnement durant le Paléolithique moyen et supérieur et le projet « d'établir la trame d'un découpage climatique au Moyen-Orient en liaison avec les géologues et les préhistoriens » (1961a, 1971,1973a, 1980b, 1984b; Solecki et Leroi-Gourhan, 1961); la paléoclimatologie du Tardiglaciaire et du début de l'Holocène (1974a, 1974b, 1980c); les prémices de l'agriculture avec l'introduction dès 1974 de la notion de proto agriculture des céréales (1969, 1974a, 1979, 1980c, 1981; Leroi-Gourhan et al., 1980).

Parallèlement à ces principaux axes de recherche, Arlette Leroi-Gourhan s'intéresse à bien d'autres sujets. Son intérêt pour l'Art pariétal, manifesté au travers de l'étude de plusieurs grottes ornées (Lascaux, Enlène, la Vache, le Portel) est couronné par la publication de Lascaux inconnu en 1979 (LeroiGourhan et Allain [dir.], 1979). Cet ouvrage rassemble des données indispensables à la compréhension du site avec une approche environnementaliste. Enfin, sa démarche paléoethnobotanique, développée très tôt (1966c) la conduit à assurer quelques analyses particulièrement prestigieuses telles la sépulture du Néanderthalien IV de Shanidar (1961a, 1968b, 1975, 1999, 2000) ou celle de la momie de Ramsès II (1985).

Outre cette production scientifique intense, Arlette LeroiGourhan s'est également attachée à l'enseignement de l'analyse pollinique. Bien des palynologues français, la plupart internationalement reconnus, ont été formés au laboratoire du musée de l'Homme (Michel Girard, Josette RenaultMiskovsky, Isabelle Roux, Aline Emery-Barbier, Anaïs 
Boyer-Klein, Gérard Firmin, Chantal Leroyer, Dominique Marguerie, Francine Darmon, Claudine Schutz, Anne di Piazza...). De surcroitt, son laboratoire a accueilli de nombreux stagiaires français ou étrangers.

Ces cinquante années de recherche seront concrétisées par plus de 170 publications $^{1}$. Elles ont également été ponctuées par la participation à de nombreux colloques, congrès et excursions géologiques, notamment organisées par l'AFEQ en France et par l'APLF et l'INQUA au niveau international; autant d'occasions pour établir des liens scientifiques et amicaux avec des préhistoriens, des géologues, des palynologues et des physiciens. En effet, l'étroite collaboration qu'Arlette Leroi-Gourhan a su établir avec les préhistoriens et les géologues l'a conduite à s'investir dans la vie associative de l'AFEQ, de l'INQUA mais surtout de la Société préhistorique française dont elle fût élue Présidente en 1971.

La reconnaissance de l'œuvre d'Arlette Leroi-Gourhan a été marquée par son entrée dans l'" Ordre du Mérite ", la médaille de chevalier lui ayant été remise le 9 mars 1990 au musée de l'Homme par le professeur Yves Coppens. Sa vie professionnelle a indéniablement été imprégnée de la personnalité de son époux mais elle a mené par elle-même une carrière remarquable, assurant la promotion des démarches naturalistes dans l'étude des hommes de la préhistoire, étendant son influence intellectuelle aussi bien en formant des palynologues qu'en tissant des liens avec la communauté scientifique internationale. Elle n'a cependant jamais sollicité l'emploi auquel elle aurait pu légitimement prétendre et est restée "bénévole " toute sa vie, continuant à plus de soixante-quinze ans à s'occuper de «son » Laboratoire de palynologie et paléobotanique au musée de l'Homme. Jusqu'en 2001, elle y conservera un petit bureau, ayant confié sa succession à Aline Emery-Barbier, lors du transfert du laboratoire d'Ethnologie préhistorique, créé en 1947 par son époux, dans les nouveaux locaux de la Maison de l'Archéologie et de l'Ethnologie à Nanterre.

Très éprouvée physiquement depuis 2002, ne se déplaçant plus qu'avec difficulté, elle s'installe à Vermenton où elle s'éteint trois ans plus tard, à l'âge de 92 ans, entourée de ses enfants.

\section{Bibliographie}

Leroi-Gourhan, A., 1956a. Analyse pollinique et Carbone 14, Bulletin de la Société préhistorique française, t. 53, fasc. 5-6, p. 291-301.

1. La liste complète des publications d'Arlette Leroi-Gourhan peut-être consultée dans le Bulletin de la Société préhistorique française, t. 103, $\mathrm{n}^{\circ} 4$, oct.-nov. 2006, p. 827-831.
Leroi-Gourhan, Arl., 1956b. Notes sur l'analyse pollinique des sédiments quaternaires des grottes, $\mathrm{XXII}{ }^{\mathrm{e}}$ Congrès préhistorique de France, Poitiers. Société préhistorique française, p. 671-675.

Leroi-Gourhan, Arl., 1957. Note sur les possibilités qu'apporte l'analyse pollinique aux études climatologiques en Afrique du Nord, Bulletin de la Société préhistorique française, t. 54, fasc. 9, p. 524-525.

Leroi-Gourhan, Arl., 1958. Résultats de l'analyse pollinique du gisement d'El Guettar (Tunisie), Bulletin de la Société préhistorique française, t. 55, fasc. 9, p. 546-551.

Leroi-Gourhan, Arl., 1959. Résultats de l'analyse pollinique de la grotte d'Isturitz, Bulletin de la Société préhistorique française, t. 56, fasc. 9-10, p. 619-624.

LeroI-Gourhan, Arl., 1960. Flores et climats du Paléolithique récent, Congrès préhistorique de France, Monaco, 1959, p. 808-813.

Leroi-Gourhan, Arl., 1961a. Analyse pollinique de la grotte de Shanidar, INQUA, VIth Congress, p. 141-143.

Leroi-Gourhan, Arl., 1961b. Flores quaternaires françaises, Bulletin de la Société Botanique de France, t. 108, fasc. 5-6, p. 244-254.

Leroi-Gourhan, Arl., 1962. La grotte de Lascaux, Bulletin de la Société Botanique de France, 88 Session extraordinaire, 1960, t. 109, p. 91-95.

Leroi-Gourhan, Arl., 1963. Archéologie et botanique, in : Courbin dir., Etudes archéologiques, SEVPEN, p. 119-125.

Leroi-Gourhan, Arl., 1965a. Les analyses polliniques sur les sédiments des grottes, Bull. de l'AFEQ, 2, p. 145-152.

Leroi-Gourhan, Arl., 1965b. Le climat Würmien pendant le Paléolithique supérieur de 36000 à 8000 B.C., Congrès de l'INQUA, Boulder USA, 1965, dactyl., 1 p. + fig.

Leroi-Gourhan, Arl., 1966a. Analyse pollinique de la Cueva del Otero, Excavaciones Arqueologica en España, t. 53, p. 83-85.

Leroi-Gourhan, Arl., 1966b. Problèmes chronologiques : chronologie botanique et chronologie absolue, La Préhistoire, vol. 1, Paris, PUF, coll. « Nouvelle Clio », p. 305-315.

Leroi-Gourhan, Arl., 1966c. L'analyse pollinique des coprolithes, Bulletin de la Société préhistorique française, t. 63, fasc. 5, p. CLXIII-CLXIV.

Leroi-Gourhan, Arl., 1967a. Pollens et datation de la grotte de la Vache (Ariège), Bulletin de la Société préhistorique de l'Ariège, t. XXII, p. 115-127.

Leroi-Gourhan, Arl., 1967b. Analyse pollinique des niveaux paléolithiques de l'abri Fritsch, Review of Palaeobotany and Palynology, Amsterdam, 4, p. 81-86.

Leroi-Gourhan, Arl., 1967c. Le Badegoulien de l'abri Fritsch : climat et chronologie, Bulletin de la Société préhistorique française, t. 64, fasc. 1, p. 95-99.

Leroi-Gourhan, Arl., 1968a. Dénomination des oscillations würmiennes. Bulletin de l'Association française pour l'étude du Quaternaire, 4, p. 281-287. 
Leroi-Gourhan, Arl., 1968b. Le Néanderthalien IV de Shanidar, Bulletin de la Société préhistorique française, t. 65, fasc. 3, p. 79-83.

Leroi-Gourhan, Arl., 1969. Pollen grains of Gramineae and Cerealia from Shanidar and Zawi Chemi, in The domestication and exploitation of plants and animals, Peter J. Ucko and G. W. Dimbleby editors, Gerald Duckworth \& CO LTD, London, p. 143-148.

Leroi-Gourhan, Arl., 1971. Middle-East Upper Quaternary Pollens. Résumé des communications section 7 : Palynology of Pliocene, Pleiostocene and Holocene, III Congress of Palynology, Novosibirsk, dactyl. 1 p.

Leroi-Gourhan, Arl., 1973a. Les possibilités de l'analyse pollinique en Syrie et au Liban, Paléorient, vol. 1, p. 39-47.

Leroi-Gourhan, Arl., 1973b. Analyses polliniques, Préhistoire et variations climatiques quaternaires, in Les méthodes quantitatives d'étude des variations du climat au cours du Pléistocène, Colloque international CNRS, n 219 , Gif-sur-Yvette, 5-8 juin 1973, p. 61-66.

Leroi-Gourhan, Arl., 1974a. Études palynologiques des derniers 11000 ans en Syrie semi-désertique, Paléorient, 2/2, p. 443-451.

Leroi-Gourhan, Arl., 1974b. Vegetational history in S.-W. Syria and Lebanon during the upper quaternary, Symposium on late Quaternary vegetational developments in extra European Aeras, Birbal Sahni Institut of Paleobotany, special publication, $n^{\circ}$ 5, p. 14-15.

Leroi-Gourhan, Arl., 1975. The flowers found with Shanidar IV, a Neanderthal burial in Irak, Science, 7 nov. 1975, vol. 190, p. 562-564.

Leroi-Gourhan, Arl., 1979. Analyse pollinique à Tell Aswad, Paléorient, vol. 5, p. 170-176.

Leroi-Gourhan, Arl., 1980a. Interstades au Würm supérieur, Supplément au Bulletin de l'Association française pour l'étude du Quaternaire, Colloque de Dijon, p. 192-193.

Leroi-Gourhan, Arl., 1980b. Les analyses polliniques au MoyenOrient, Paléorient, vol. 6, p. 79-91.

Leroi-Gourhan, Arl., 1980c. Analyse pollinique de Zawi Chemi, in An early village site at Zawi Chemi-Shanidar, R. L. Solecki dir., Bibliotheca mesopotamica, vol. 13, p. 77-79.

Leroi-Gourhan, Arl., 1981. Palynology and the beginning of agriculture in the Near East, IV Int. Palynol. Conf., Lucknow (1976-1977), 3, p. 258-262.

Leroi-Gourhan, Arl., 1984a. Paysages et climats il y a 35000 ans, Les Dossiers Histoire et Archéologie, n 87, p. 20-24.

Leroi-Gourhan, Arl., 1984b. L'évolution de la Végétation au Moyen-Orient lors des derniers 100000 ans, Webbia, 38, p. $455-463$.

Leroi-Gourhan, Arl., 1985. Les pollens et l'embaumement, in L. Balout, C. Roubet (dir.), La momie de Ramsès II : recherches sur les civilisations, Paris, ADPF, p. 162-165.

Leroi-Gourhan, Arl., 1989a. Faunes et flores, Le temps de la Préhistoire, vol. 2, Société Préhistorique française et Archéologia, p. 54-59.
Leroi-Gourhan, Arl., 1989b. Stratigraphie du Würm depuis 35000 ans par la palynologie, Type Sections : Imagination or Reality? Rose \& Schlüder (eds), Quaternar, Balkema, Rotterdam, p. 91-92.

Leroi-Gourhan, Arl., 1992. Bois et pollens : études complémentaires, Bulletin de la Société Botanique de France, 139, Actual. bot. (2), p. 273-280.

Leroi-Gourhan, Arl., 1997. Chauds et Froids de 60000 à 15000 B.P., Bulletin de la Société préhistorique française, t. 94, fasc. 2, p. 151-160.

Leroi-Gourhan, Arl., 1999. Shanidar et ses fleurs. Paléorient, 24/2, 79-88.

Leroi-Gourhan, Arl., 2000. Rites et langage à Shanidar? Bulletin de la Société préhistorique française, t. 97, fasc. 2, p. 291-293.

Leroi-Gourhan, Arl., Allain, J. et al., 1979. Lascaux inconnu, XII ${ }^{\mathrm{e}}$ supplément à Gallia-Préhistoire, $381 \mathrm{p}$.

Leroi-Gourhan, Arl., Girard, M., 1976. Palynologie et Chronologie au Paléolithique supérieur et à l'Épipaléolithique en Europe de l'Ouest, IX Congrès UISPP, Nice, Résumé des communications, 1 carte, 4 tableaux, p. 71.

Leroi-Gourhan, Arl., Girard, M., 1979. Chronologie pollinique de quelques sites préhistoriques à la fin des temps glaciaires, in La fin des temps glaciaires en Europe, Colloque CNRS n ${ }^{\circ} 271$, Talence, mai 1977, p. 583-588.

Leroi-Gourhan, Arl., 1965. Chronologie des grottes d'Arcysur-Cure (Yonne), I. Climats du Quaternaire récent, GalliaPréhistoire, t. VII, 1964, p. 1-35.

Leroi-Gourhan, Arl., Leroi-Gourhan, A., 1989. Un voyage chez les Aïnous, Paris, Albin Michel, 156 p.

Leroi-Gourhan, Arl., Noy, T., Schuldenrein, J., Tchernov, E., 1980. Gilgal, a Pre-Pottery Neolithic A site, in the Lower Jordan Valley, Israel Exploration Journal, 30, p. 63-82.

Leroi-Gourhan, Arl., Renault-Miskovsky J., 1977. La palynologie appliquée à l'Archéologie, in Approche écologique de l'Homme fossile, chap. 3, Paléobotanique, supplément au Bulletin de l'Association française pour l'étude du Quaternaire, t. 47, 35-49.

Leroi-Gourhan, Arl., YamanaKa, I., 1991. Un voyage chez les Aïnous, traduction en japonais, Tokyo, $172 \mathrm{p}$.

Renault-Miskovski J., Leroi-Gourhan, Arl., 1981. Palynologie et Archéologie : nouveaux résultats du Paléolithique supérieur au Mésolithique, Bulletin de l'Association française pour l'étude du Quaternaire, fasc. 3-4, p. 121-128.

Solecki R., Leroi-Gourhan, Arl., 1961. Palaeoclimatology and archaeology in the Near-East, Annals of the New York Academy of Sciences, vol. 95, article 1, p. 729-739.

Van Campo M., Leroi-Gourhan, Arl., 1956a. Un paysage forestier rissien dans l'Yonne, Bulletin de la Société botanique de France, 103, n ${ }^{\circ}$ 5-6, p. 285-286.

Van Campo M., Leroi-Gourhan, A., 1956b. Note préliminaire à l'étude des pollens fossiles de différents niveaux des grottes d'Arcy-sur-Cure, Bulletin du Museum, 2e série, t. XXVIII, 3, p. 326-330. 